\title{
Autologous stem cell transplantation in patients with extragonadal germ cell tumors: A single center experience
}

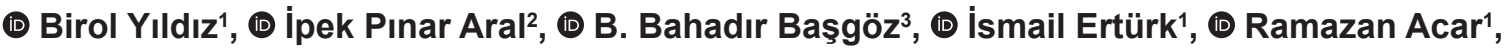 \\ (1) Nuri Karadurmuş ${ }^{1}$
}

1 University of Health Sciences Turkey, Gülhane Training and Research Hospital, Clinic of Medical Oncology, Ankara, Turkey

2Nevsehir State Hospital, Clinic of Radiation Oncology, Eskisehir, Turkey

3University of Health Sciences Turkey, Gülhane Training and Research Hospital, Clinic of Internal Medicine, Ankara, Turkey

Date submitted:

26.11.2019

Date accepted:

23.12.2019

Online publication date:

15.06.2020

\section{Corresponding Author:}

Birol Yıldız MD, University of Health

Sciences Turkey, Gülhane Training

and Research Hospital, Clinic of

Medical Oncology, Ankara, Turkey

bfyildiz@gmail.com

ORCID:

orcid.org/0000-0001-8920-6467

Presented in: 3rd Immunotherapy and Oncology Congress 2019/Antalya.

Keywords: Autologous stem cell transplantation, extragonadal germ cell tumors, salvage treatment

\section{ABSTRACT}

Aims: In extragonadal germ cell tumors (EGGCT), chemosensitivity and prognosis are worse than in GCT tumors. There is no standard salvage chemotherapy regimen for relapsed/ refractory EGGCT patients. Autologous stem cell transplantation (ASCT) has increasingly been used in recent years as a second or third salvage therapy. In this study, we aimed to share our experience with the relapsed/refractory EGGCT patients who underwent ASCT.

Methods: The data of patients who underwent ASCT and were followed up according to tango in EGGCT between 1991 and 2015 at the University of Health Sciences Turkey, Gülhane Training and Research Hospital, Ankara, Turkey, were retrospectively analyzed.

Results: This study included 30 patients. The most common involvement site was the retroperitoneum $(n=20,66.7 \%)$, followed by the mediastinum $(n=10,33.3 \%)$. Retroperitoneal lymph node dissection was performed in 14 patients. Due to relapse, 27 patients received 3 cycles of TIP (paclitaxel, ifosfamide and cisplatin) and three patients received 3 cycles of VIP (etoposide, ifosfamide and cisplatin) treatment as the first salvage. Complete response was achieved in 10 patients. Progression was recorded in five patients. As the second salvage, 13 patients received VIP and three patients received TIP. In addition, 10 patients underwent ASCT as the second salvage and 20 patients had the third salvage. Nine patients died and 21 patients were still under follow-up.

Conclusions: Although the chemosensitivity and prognosis are worse in patients with EGGCT than in those with gonadal GCT, their survival seems to be increased significantly with multimodal treatments and ASCT.

\section{Introduction}

Testicular cancer is the most common solid malignancy in men aged $15-35$ years, $95 \%$ of which are germ cell tumors (GCT) (1). GCT is primarily located in the gonadal region and rarely occurs in an extragonadal area such as the mediastinum and retroperitoneum. Extragonadal GCT (EGGCT) are formed by malignant transformation of gonadal cells that have not completed their migration during embryogenesis without a gonadal primary mass in ultrasonographic evaluation (2). EGGCT accounts for $2-5 \%$ of all GCT and has an approximate incidence of $1 / 1,000,000$ (3).
Although EGGCT has similar histological, serological and cytogenetic characteristics as gonadal GCT, their behavior is different clinically and biologically. EGGCT has a worse chemosensitivity and prognosis than gonadal tumors $(4,5)$. The treatment of EGGCT patients is similar to the treatment of gonadal GCT, and after histological separation as seminoma and non-seminoma, chemotherapy (CT) is applied according to risk classification. Surgical resection is also performed in patients with residual tumors. This multimodal treatment increases efficacy and survival $(6,7)$. There is no standard salvage CT treatment for relapsed/refractory EGGCT patients. Autologous 
stem cell transplantation (ASCT) therapy for EGGCT patients has started to increase gradually and is mostly applied as second or third salvage (8).

The aim of this study was to share our single center experience with patients who underwent salvage ASCT with the diagnosis of relapse/refractory EGGCT.

\section{Methods}

In this study, 30 patients with EGGCT who underwent high dose CT (HDCT) and ASCT between 4 February 1991 and 12 May 2015 at the University of Health Sciences, Gülhane Training and Research Hospital, Medical Oncology Clinic, Ankara, Turkey were evaluated retrospectively. The study was approved by the Ethics Committee of Gülhane Training and Research Hospital with the decision number 18/159. Patient interview information, patient files and electronic system data were used to obtain data. Patients' demographic status, tumor localization, first recurrence dates, salvage CT protocols and treatment responses, HDCT and ASCT time, and HDCT and ASCT recurrence and final status were noted.

The primary endpoint is overall survey (OS) and progression free survey (PFS). The diagnosis date was accepted as the start date for the general OS value of the patients, the end point was the last control date for the surviving patients, and the exitus date for the ex-patients. In order to calculate OS values after HDCT and ASCT, start date HDCT and ASCT date is accepted. Endpoint for $O S$ is the last control date for living patients, exitus date for ex patients.

The PFS values of the patients after the first step treatment were calculated as PFS1. In addition, the time to recurrence after HDCT and ASCT was calculated as PFS2; HDCT and ASCT dates were taken as the starting date, relapse date for the relapse as the endpoint, and the last control date for the non-relapse.

Patients with pathologic evidence of EGGCT who had relapsed after first-line treatment and who had HDCT and ASCT were included in the study. Patients who did not have HDCT and ASCT and whose files and follow-up information were missing were excluded from the study.

\section{Statistical Analysis}

Statistical Package for Social Sciences version 24.0 was used for conducting statistical analysis of data (IBM Corp, Armonk, NY, USA). Descriptive statistics for expressing continuous (quantitative) variables were mean, standard deviation, minimum and maximum values, while the categorical variables were expressed as number $(n)$ and ratio (\%). The suitability of the variables to the normal distribution was evaluated by visual and analysis methods and nonparametric tests were used because they did not fit the normal distribution. The chi-square and Fisher's exact tests were used to determine the demographic characteristics of the patients. The KaplanMeier was used for univariate survey analysis and log rank test was used. In multivariate analyses, the Cox regression test was used. The Spearman's rank correlation test was used for univariate correlation analysis. Statistically significant value was accepted as that less than 0.05 .

\section{Results}

A total of 30 patients who underwent HDCT and ASCT were included in the study. The median age of the patients was 41 years (range: 21-60). First line 4 cycles of BEP (bleomycin, etoposide, cisplatin) were applied to all patients. During followup, all patients relapsed and the median PFS1 was 16 (range: 3-45) months. TIP (paclitaxel, ifosfamide and cisplatin) was given to $27(90 \%)$ patients after the relapse and VIP (etoposide, ifosfamide and cisplatin) salvage CT was applied to $3(10 \%)$ patients. After Salvage CT, complete response (CR) in 10 (33.3\%), partial response (PR) in $12(40 \%)$, stable response in three $(10 \%)$ and progression in five $(16.7 \%)$ patients were observed. A total of 20 (66.7\%) patients who did not receive CR underwent second salvage CT. Four (20\%) of the patients who underwent second salvage CT were treated with TIP; 16 patients (80\%) received VIP treatment. In 6 (33.3\%) CR, 9 (49\%) PR and $3(17.7 \%)$, stable responses were observed in the second salvage CT. The primary characteristics were summarized in Table 1.

\begin{tabular}{|lll|}
\hline Table 1. Primary characteristics \\
\hline Primary site, $\mathrm{n}(\%)$ & Retroperitoneal & $20(66.7)$ \\
\hline & Mediastinal & $10(33.3)$ \\
\hline Pathology, $\mathrm{n}(\%)$ & Embryonal carcinoma & $3(10)$ \\
\hline & Teratoma & $10(33.3)$ \\
\hline & Mixed nonseminoma & $8(26.7)$ \\
\hline & Choriocarcinoma & $7(23.3)$ \\
\hline & Yolk sac & $2(6.7)$ \\
\hline Metastasis, $\mathrm{n}(\%)$ & Yes & $16(53.3)$ \\
\hline & No & $14(46.7)$ \\
\hline Metastasis-site, $\mathrm{n}(\%)$ & Brain & $4(25)$ \\
\hline & Bone & $1(6.3)$ \\
\hline & Lung & $7(43.8)$ \\
\hline & Liver & $1(6.3)$ \\
\hline & Multiple organs & $3(18.8)$ \\
\hline RPLND, $\mathrm{n}(\%)$ & Yes & $14(46.7)$ \\
\hline & No & $16(53.3)$ \\
\hline $\begin{array}{l}\text { RPLND pathology, } \mathrm{n} \\
(\%)\end{array}$ & Necrosis & $7(50)$ \\
\hline & Viable tm & $7(50)$ \\
\hline $\begin{array}{l}\text { Metastasectomy, } \mathrm{n} \\
(\%)\end{array}$ & Yes & $3(10)$ \\
\hline & No & $27(90)$ \\
\hline & &
\end{tabular}




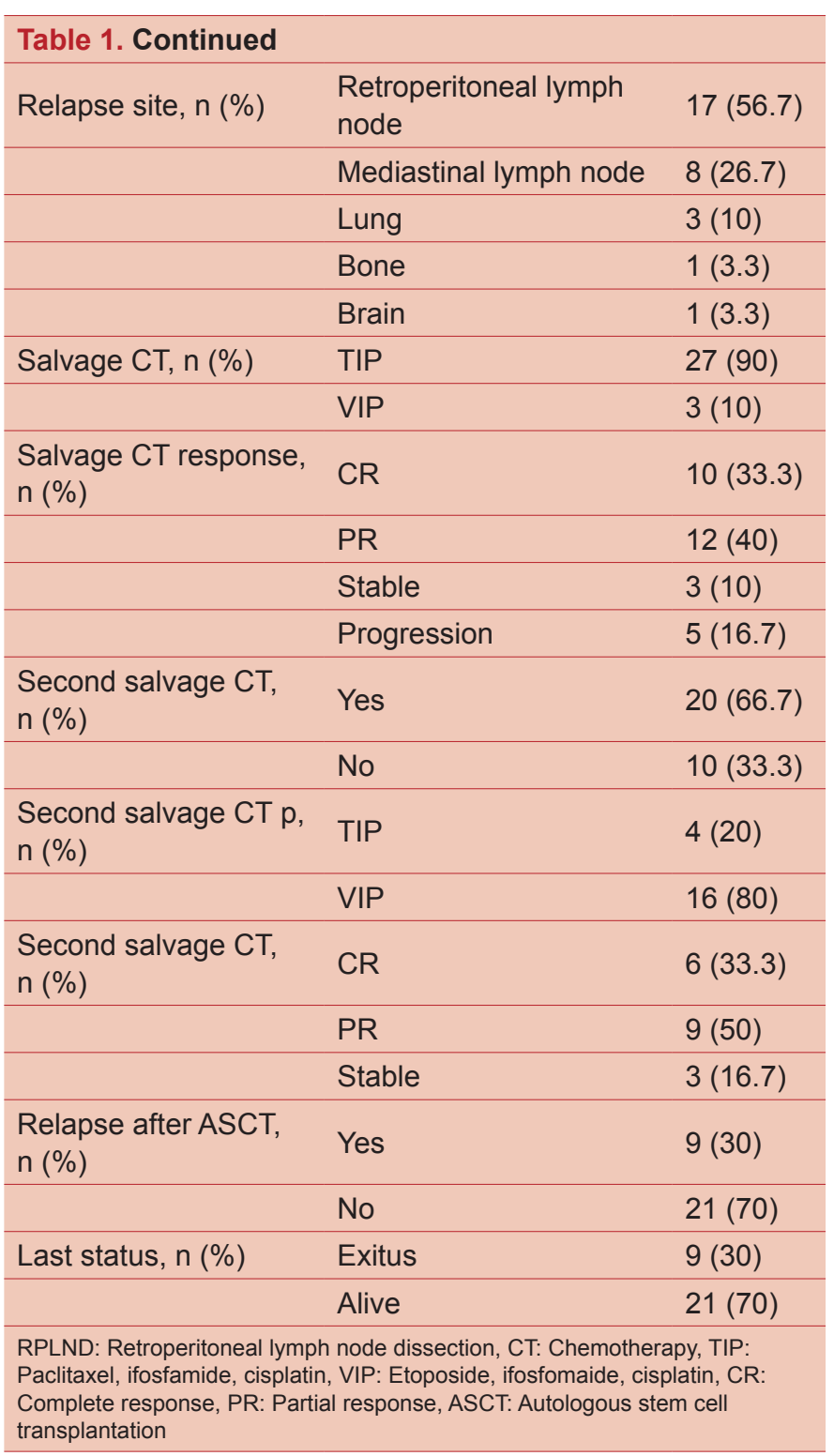

All patients underwent HDCT and ASCT between 4 February 1991 and 12 May 2015. In our study, the follow-up period after diagnosis was 137 months (range: 30-353 months); the median follow-up period after HDCT and ASCT was 110 months (range: 29-327 months).

Patients who relapsed after the first step treatment were included in the study. The median PFS1 value of patients until the first relapse after the first cure was 16 months (range: 3-45 months). The patient relapsed for 9 months (30\%) after HDCT and ASCT. The median value of PFS2 after HDCT and ASCT is 101 months (range: 26-235 months). All patients who recurred after HDCT and ASCT were ex.

According to our current data, $9(30 \%)$ patients died and 21 (70\%) patients were alive. The median OS $_{\text {diagnosis }}$ value after diagnosis was 136 months (range: 31 to 353 months), and the median OS $_{\text {ASCT }}$ value after HDCT and ASCT was 103 months (range: 29-327 months).

The 2-year $\mathrm{OS}_{\text {diagnosis }}$ value of the patients in our study group was $97.6 \%$; the 5 -year OS $_{\text {diagnosis }}$ value was $93.2 \%$ and the 10 -year OS $_{\text {diagnosis }}$ value was $84.7 \%$. The 2 -year OS ASCT $_{\text {ASC }}$ value of our patients was $93.7 \%$; the 5 -year OS $_{\text {ASCT }}$ value was $88.4 \%$ and the 10 -year OS $_{\text {ASCT }}$ value was $78.3 \%$. The 2 -year PFS $_{\text {ASCT }}$ value of our patients was $92.6 \%$; the 5 -year PFS ASCT $_{\text {. }}$ value was $87 \%$ and the 10 -year $\mathrm{PFS}_{\mathrm{ASCT}}$ value was $77.5 \%$.

\section{Factors affecting $\mathrm{OS}_{\text {diagnosis }}$}

The median $\mathrm{OS}_{\text {diagnosis }}$ value of the patients after diagnosis was 136 (range: 31 to 353) months. The mediastinal or retroperitoneal (RP) localization of the disease did not affect $\mathrm{OS}_{\text {diagnosis }}$ significantly $(\mathrm{p}=0.072)$. While the median $\mathrm{OS}_{\text {diagnosis }}$ was found to be 140 months (range: $30-319)$ in patients with RP, the median was 133 months (66-353) in those with the mediastinum (Figure 1).

When the relationship between the pathologies and OS $_{\text {diagnosis }}$ was evaluated, the median was 186 months (range: 118-223) for embryonic carcinoma, the median was 136 months for teratoma (range: 30-218), the median was 130 months (range: 51-319) for mixed nonseminoma, the median was 107 months (range: 66-162) for patients with choriocarcinoma; the median was 222 months (206-240) in the yolk sac.

$\mathrm{OS}_{\text {diagnosis }}$ was significantly affected by the relapse region of the patients $(p=0.001)$. The median OS diagnosis was 135 months (range: 51-240) in patients with relapse RP, and 150 months (range: 86-353) in patients with recurrent mediastinal region, 118 months (range: 66-218) in patients with recurrence of the lung, 30 months only in the case of recurrence in the bone and 319 months in the only case of recurrence in the brain. When the subgroup analysis was performed, it was found that the relapse in the bone where the significance was caused by bone had a significantly lower OS $_{\text {diagnosis }}$ value than the others.

There was a significant relationship between recurrence

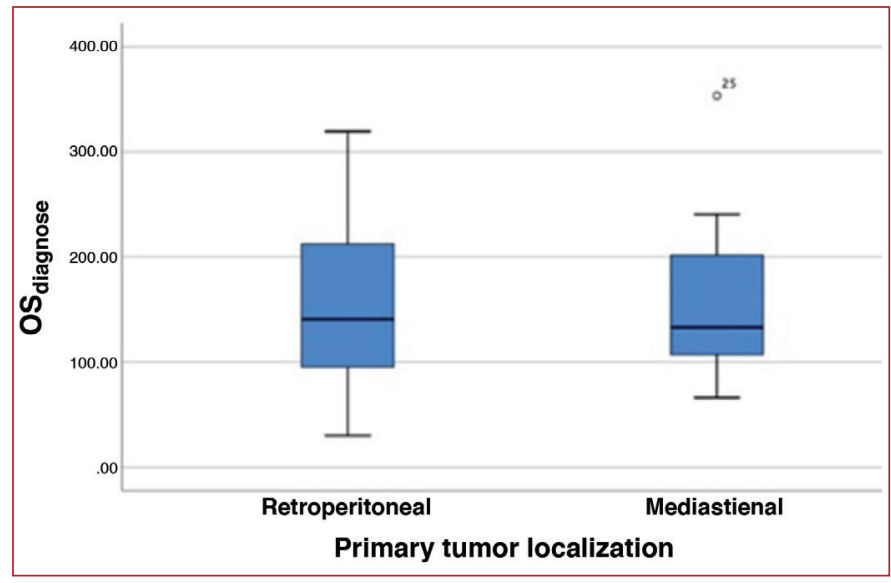

Figure 1. Relationship between $\mathrm{OS}_{\text {diagnose }}$ and primary tumor localization OS $_{\text {diagnose: }}$ Overall survival from the time of diagnosis 
after HDCT and ASCT and $\mathrm{OS}_{\text {diagnosis }}(\mathrm{p}=0.001)$. The median value of OS $_{\text {diagnosis }}$ after HDCT and ASCT was 136 months (range: 51-353) and the median OS $_{\text {diagnosis }}$ value was 130 months (range: $30-240$ ).

\section{Factors affecting $\mathrm{OS}_{\mathrm{ASCT}}$}

The median $\mathrm{OS}_{\mathrm{ASCT}}$ value after the ASCT date was 103 (range: 29-327) months. Primary mediastinal or RP did not significantly affect $\mathrm{OS}_{\mathrm{ASCT}}(\mathrm{p}=0.075)$. While the median OS ASCT was 101 months (range: 29-304) in patients with RP and 103 months (49-327) in patients with primary mediastinum.

OS $_{\text {ASCT }}$ was found to have a significant effect on the relapse region $(p=0.006)$. The median time was 114 months (range: 51 304 ) in patients with relapse RP and 90 months (range: 36-327) in patients with mediastinal area, 80 months (range: 45-153) in patients with lung recurrence, 29 months in a single case with bone and 50 months in a single case of recurrence in the brain. When the subgroup analysis was performed, it was found that the bone recurrent case had a significantly lower $\mathrm{OS}_{\mathrm{ASCT}}$ value than the others (Figure 2).

When the relationship between $\mathrm{OS}_{\mathrm{ASCT}}$ and the pathology of the patients was evaluated, the median was 172 months for embryonal carcinoma (range: 80-202), 109 months (range: 29-153) for teratoma, 88 months (range: 45-304) for mixed nonseminoma, 48 months (range: $36-117$ ) for choriocarcinoma

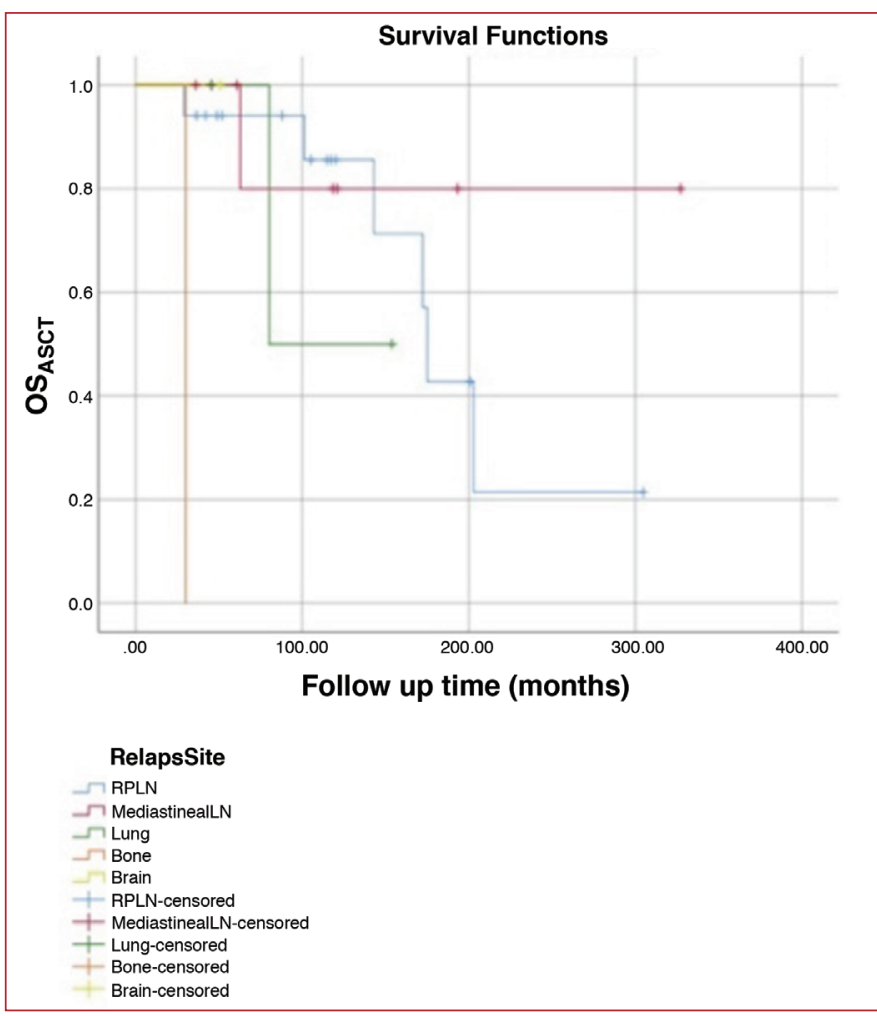

Figure 2. Relation of $\mathrm{OS}_{\mathrm{ASCT}}$ to relapse site

${ }_{O S} S_{A S C T}$ : Overall survival from the time of autologous stem cell transplantation, RPLN: Retroperitoneal lymph node, Mediastinal LN: Mediastinal lymph node and 197 months (143-200) for yolk sac.

Unlike $\mathrm{OS}_{\text {diagnosis}}, \mathrm{OS}_{\mathrm{ASCT}}$ was significantly affected by the age of the patients. The median OS $_{\text {ASCT }}$ value of patients aged 40 years and under was 47 months (range: 28-118), whereas for those over 40 years, this value was 148 months (range: $50-327$ ) $(p=0.012)$ (Figure 3).

\section{Factors affecting PFS2 $\left(\right.$ PFS $\left._{\mathrm{ASCT}}\right)$}

The median value of PFS2 (PFS $A S C T)$ after HDCT and ASCT was 101 months (range: 26-235). All patients who recurred after HDCT and ASCT died. PFS2 did not significantly affect the mediastinal or RP primer $(\mathrm{p}=0.070)$. While the median PFS2 was 101 months (range: 26-304) in patients with RP primer, the median PFS2 was 101 months (45-327) in those with mediastinal primer.

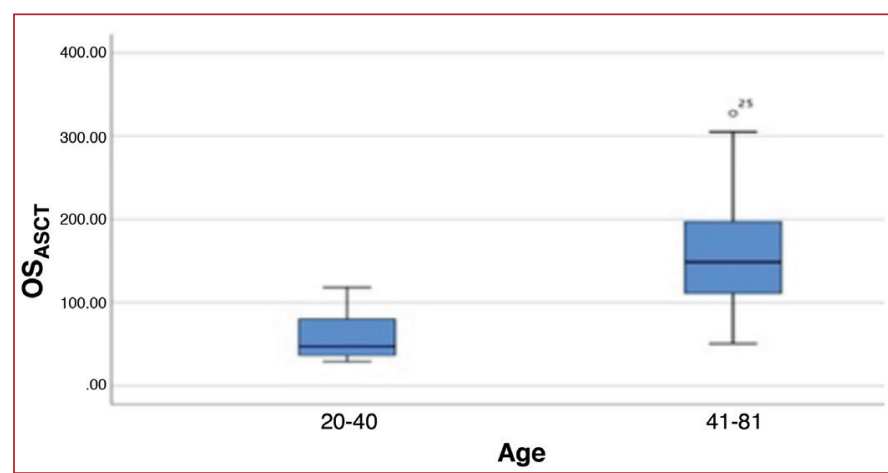

Figure 3. Evaluation of the relationship between $\mathrm{OS}_{\mathrm{ASCT}}$ and age OS $_{\text {ASCT: }}$ Overall survival from the time of autologous stem cell transplantation, RPLN: Retroperitoneal lymph node, Mediastinal LN: Mediastinal lymph node

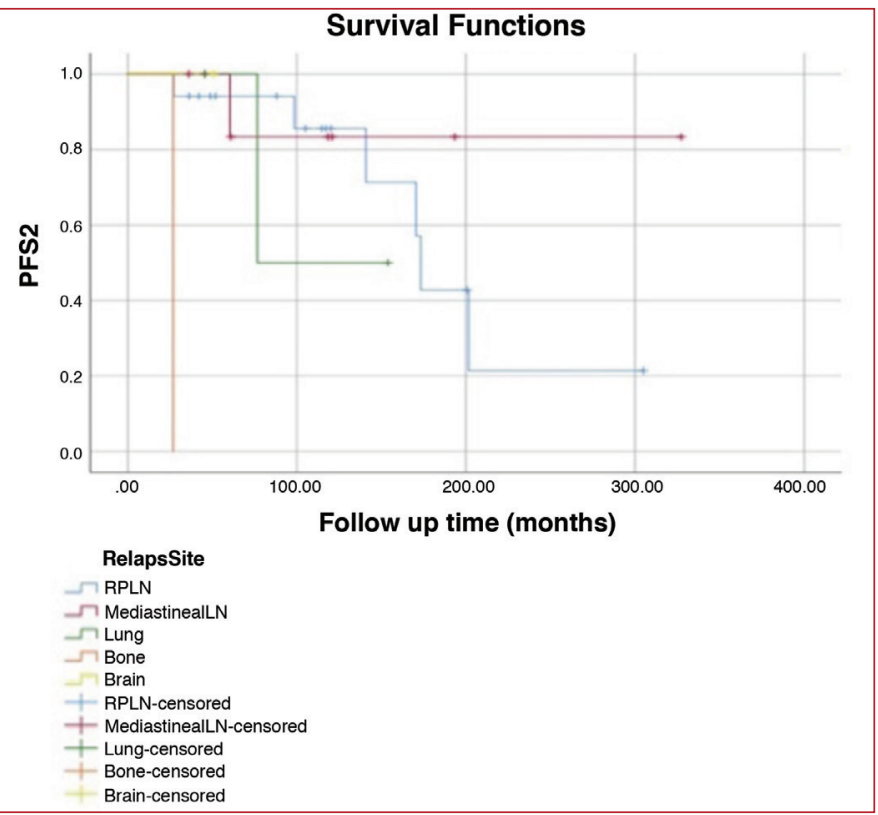

Figure 4. The relationship between PFS2 and relapse site

PFS2: Progression free survival from the time of autologous stem cell transplantation, RPLN: retroperitoneal lymph node, Mediastinal LN: Mediastinal lymph node 
The relapse region of the patients was significantly affected by PFS2 ( $p=0.001)$. the median PFS2 was 112 months (range: 27-27304) in patients with relapse RP, and 86 months (range: 35327 ) in patients with mediastinal recurrence. It was 76 months (range: 40-153) in patients with lung recurrence, 26 months in bone recurrence, and 48 months in single brain recurrence. When subgroup analysis was performed, it was found that the recurrent bone case had a significantly lower PFS2 value than the others (Figure 4).

When the relationship between PFS2 and patients' pathology was evaluated, it was found that the median PFS2 was 170 months (range: 76-201) in embryonal carcinoma, 109 months in teratoma (range: 26-153), 88 months in mixed nonseminoma (range: 43-304), 48 months in choriocarcinoma (range: 36-117) and 197 months (143-200) in the yolk sheet.

PFS2 was significantly affected by the age of patients at the time of ASCT. The median PFS2 value was 45 months (range: 26-118) for patients aged 40 years and younger, and 147 months (range: 48-327) for those over 40 years of age $(p=0.010)$.

\section{Discussion}

In relapsed/refractory GCT patients, HDCT and ASCT have been used as standard salvage treatment and are often used for second or third salvage purposes (9). However, due to the low number of relapsed/refractory EGGCT patients, the efficacy of HDCT and ASCT in these patients could not be clearly demonstrated due to the lack of randomized trials. In this study, we evaluated the demographic characteristics, progressionfree and total survival data retrospectively in our center to demonstrate the efficacy of treatment in relapsed/refractory EGGCT patients who underwent HDCT and ASCT for second or third salvage purposes.

All of our EGGCT patients had histopathology in the non-seminomatous group and all of them were in the poor prognostic group according to IGCCG $(9,10)$. We performed HDCT and ASCT as the second salvage in 10 of our patients and as the third salvage in 20 of our patients. There was no significant relationship between salvage application step and OS and PFS. There is a high chance of success in the studies performed in GCT patients in the third step and before, and it provides duration of response even in the next steps (11).

In many studies, VIP as the first salvage regimen is preferred primarily $(12,13)$, but in our patients we preferred TIP treatment as the first salvage, as in the study of 69 patients included by Ko et al. (14). There is no study designed to compare the advantages of TIP and VIP regimens as salvage treatment.

Total survival in EGGCT patients varies according to primary tumor localization, relapse status, relapse duration and IGCCCG criteria. While 5 -year OS is $65 \%$ in primary RP
EGGCT, this rate decreases to $40-45 \%$ in mediastinal EGGCT patients $(2,12,15,16)$. In a study conducted by Schmoll et al. (17), the disease-specific survival of RP EGGCT patients who underwent HDCT and ASCT was reported to be $76 \%$. In our study, the 5-year OSASCT value was reported as $88.4 \%$ and clearly shows the efficacy of the treatment.

In our study, the mean age was 41 years and 15 years older than the median age in the literature $(18,19)$. The average age is high compared to the literature, decreases bone marrow reserve, causes neutropenia and febrile neutropenia complications, and may lead to an increase in mortality rate. In addition, in the literature (20), the average rate of advanced disease was $30 \%$, whereas in our study, this rate was $50 \%$ and the mortality risk was high. In our study, nine patients died and despite this high risk, this rate is significantly lower than in the literature. In addition, in our study, an unspecified result was obtained in the literature and $\mathrm{OS}_{\mathrm{ASCT}}$ and PFS2 were significantly better in patients over 40 years of age, who underwent HDCT and ASCT.

Hege et al.'s (21) study revealed that treatment-related mortality was found to be $5.5 \%$ in HDCT and ASCT patients and Adra et al. (22) also presented treatment-related mortality as $2.4 \%$ (21). In our study, no patients died in association with the treatment.

Considering the limitations of our study, the study consisted of a report on rare case series that underwent AHSCT due to relapsed or refractory EGGCT; therefore, it included a small sample size of patients. Also, it had a heterogeneous patient population with regard to indications for AHSCT and it was a retrospective study.

\section{Conclusion}

As a result, for patients with relapsed/refractory EGGCT, high dose ifosfamide/carboplatin/etoposide regimen was safe and and an effective treatment choice. Although the chemosensitivity and prognosis of EGGCT patients are worse than GCT patients, their survival is significantly increased with multimodal treatments and ASCT. Future prospective randomized studies should reveal more reasonable and effective survival results.

\section{Acknowledgements}

We thank our patients and their families who participated in the research helpfully and devotedly without expecting material compensation. We are grateful to Prof. Dr. İlker Taşçı for critically editing of the manuscript.

\section{Ethics}

Ethics Committee Approval: The study was approved by the Ethics Committee of Gülhane Training and Research Hospital with the decision number 18/159.

Informed Consent: Retrospective study. 
Peer-review: Externally peer-reviewed.

\section{Authorship Contributions}

Surgical and Medical Practices: B.B.B., B.Y., R.A., İ.E., Concept: B.Y., N.K., I.P.A., Design: B.Y., N.K., İ.P.A., Data Collection or Processing: B.Y., İ.E., B.B.B., R.A., Analysis or Interpretation: I.P.A., B.Y., Literature Search: R.A., I.E., B.Y., N.K., Writing: B.B.B., R.A., B.B.B., N.K.

Conflict of Interest: No conflict of interest was declared by the authors.

Financial Disclosure: The authors declared that this study received no financial support.

\section{References}

1. Siegel RL, Miller KD, Jemal A. Cancer statistics, 2019. CA Cancer J Clin 2019;69:7-34.

2. Bokemeyer C, Nichols CR, Droz JP, et al. Extragonadal germ cell tumors of the mediastinum and retroperitoneum: Results from an international analysis. J Clin Oncol. 2002;20:1864-1873.

3. Mosbech $\mathrm{CH}$, Rechnitzer C, Brok JS, Rajpert-DeMeyts E, Hoei-Hansen CE. Recent advances in understanding the etiology and pathogenesis of pediatric germ cell tumors. J Pediatr Hematol Oncol. 2014;36:263-270.

4. Fizazi K, Culine S, Droz JP, Le Chevalier T, Ruffie P, Theodore C. Primary mediastinal non-seminomatous germ cell tumors: from clinics to biology. Bull Cancer. 1997;84:313-327.

5. Toner GC, Geller NL, Lin SY, Bosl GJ. Extragonadal and poor risk nonseminomatous germ cell tumors. Survival and prognostic features. Cancer. 1991;67:2049-2057.

6. Albers P, Albrecht W, Algaba F, et al. EAU guidelines on testicular cancer: 2011 update. Eur Urol. 2011;60:304-319.

7. Beyer J, Albers P, Altena R, et al. Maintaining success, reducing treatment burden, focusing on survivorship: Highlights from the third European consensus conference on diagnosis and treatment of germ-cell cancer. Ann Oncol. 2013;24:878-888.

8. Albany C, Einhorn LH. Extragonadal germ cell tumors: Clinical presentation and management. Curr Opin Oncol. 2013;25:261-265.

9. Kumano M, Miyake H, Hara I, Furukawa J, Takenaka A, Fujisawa M. First-line high-dose chemotherapy combined with peripheral blood stem cell transplantation for patients with advanced extragonadal germ cell tumours. Int J Urol. 2007;14:336-338.

10. Rosti G, De Giorgi U, Wandt H, et al. First-line high-dose chemotherapy for patients with poor prognosis extragonadal germ cell tumours: The experience of the European Bone Marrow Transplantation (EBMT) Solid Tumours Working Party. Bone Marrow Transplant. 2004;34:1033-1037.
11. Bokemeyer C, Droz JP, Horwich A, et al. Extragonadal seminoma: An international multicentre analysis of prognostic factors and long-term treatment outcome. Cancer. 2001;91:1394-1401.

12. Hartmann JT, Einhorn L, Nichols CR, et al. Second-line chemotherapy in patients with relapsed extragonadal nonseminomatous germ cell tumours: Results of an international multicenter analysis. J Clin Oncol. 2001;19:1641-1648.

13. De Giorgi $U$, Demirer $T$, Wandt $H$, et al. Second-line high-dose chemotherapy in patients with mediastinal and retroperitoneal primary non-seminomatous germ cell tumours: The EBMT experience. Ann Oncol. 2005;16:146151.

14. Ko JJ, Asif T, Li H, Alimohamed N, Nguyen PT, Heng DY. Disease characteristics and survival outcomes of extragonadal primary germ cell tumour in two Canadian tertiary cancer centres. Can Urol Assoc J. 2016;10:165170.

15. McKenney JK, Heerema-McKenney A, Rouse RV. Extragonadal germ cell tumours: A review with emphasis on pathologic features, clinical prognostic variables, and differential diagnostic considerations. Adv Anat Pathol. 2007;14:69-92.

16. Hsu YJ, Pai L, Chen YC, Ho CL, Kao WY, Chao TY. Extragonadal germ cell tumours in Taiwan: An analysis of treatment results of 59 patients. Cancer. 2002;95:766-774.

17. Schmoll HJ, Kollmannsberger C, Metzner B, et al. Longterm results of first-line sequential high-dose etoposide, ifosfamide, and cisplatin chemotherapy plus autologous stem cell support for patients with advanced metastatic germ cell cancer: an extended phase I/II study of the German Testicular Cancer Study Group. J Clin Oncol. 2003;21:4083-4091.

18. Gonzalez Vela JL, Villalona-Calero MA, Torkelson JL, Fraley EE, Kennedy BJ. Extragonadal abdominal germ cell cancers. Am J Clin Oncol. 1992;15:308-310.

19. Goss PE, Schwertfeger L, Blackstein ME. Extragonadal germ cell tumors. A 14-year Toronto experience. Cancer. 1994;73:1971-1979.

20. Dueland S, Stenwig A, Heilo A, Høie J, Ous S, Fosså SD. Treatment and outcome of patients with extragonadal germ cell tumours - the Norwegian Radium Hospital's experience 1979-94. Br J Cancer. 1998;77:329-335.

21. Hege SH, Anna L, Ulrika S, et al. High-dose chemotherapy with autologous stem cell support in patients with metastatic nonseminomatous testicular cancer - a report from the Swedish Norwegian Testicular Cancer Group (SWENOTECA). Acta Oncol. 2012;51;168-176.

22. Adra N, Abonour R, Althouse SK, Albany C, Hanna NH, Einhorn LH. High dose chemotherapy and autologous peripheral blood stem cell transplantation for relapsed metastatic germ cell tumors: The Indiana University Experience. J Clin Oncol. 2017;35:1096-1102. 